\title{
Charge oscillations in Quantum Dots: Renormalization group and Hartree method calculations
}

\author{
Michael Sindel, ${ }^{1}$ Alessandro Silva, ${ }^{2,3}$ Yuval Oreg, ${ }^{3}$ and Jan von Delft四 \\ ${ }^{1}$ Physics Department and Center for NanoScience, LMU München, 80333 München, Germany \\ ${ }^{2}$ Center for Materials Theory, Department of Physics and Astronomy, Rutgers University, Piscataway, NJ 08854,USA \\ ${ }^{3}$ Department of Condensed Matter Physics, The Weizmann Institute of Science, Rehovot 76100, Israel
}

(Dated: September 29, 2018)

\begin{abstract}
We analyze the local level occupation of a spinless, interacting two-level quantum dot coupled to two leads by means of Wilson's numerical renormalization group method. A gate voltage sweep, causing a rearrangement of the charge such that the system's energy is minimized, leads to oscillations, and sometimes even inversions, in the level occupations. We find that these oscillations, qualitatively understandable by a simple Hartree analysis, are generic and occur in a wide range of system parameters. By allowing a relative sign in one tunneling matrix element between dot and leads, we extend our findings to more generic models. Experimental applications and the qualitative effect of spin are discussed.
\end{abstract}

PACS numbers: 73.63.Kv, 73.23.Hk, 72.10.-d

\section{INTRODUCTION}

The Coulomb blockade $(\mathrm{CB})^{\frac{1}{1}}$ is one of the most basic and fundamental phenomena in quantum dot (QD) physics: according to the standard single-electron tunneling picture for transport through small QD's, electrons can only pass through the dot one by one if the Coulomb charging energy $U$ is the dominant energy scale. Likewise, if the gate voltage (applied to a plunger gate near the QD) is changed, resulting in a rigid shift of the single-particle spectrum of the QD relative to the Fermi energy of the leads, the single-particle levels of the QD are naively expected to be filled "one by one". Many basic single-electron devices and QD-based qubit proposals² are based on this simple picture.

In this paper we point out that under quite generic conditions, the charging process is actually more complicated, and the occupation functions for the single-particle levels can show some rather complex, non-monotonic behavior as a function of gate voltage, deviating considerably from the standard CB picture ["one by one" filling]. This complex behavior turns out to be rather generic for a QD coupled to leads that can supply the QD with electrons, and originates from the competition between the QD-leads coupling $\Gamma$, and the intrinsic energy scales of the QD, namely its charging energy $U$ and levels spacing $\Delta$.

In order to study this competition, it suffices to consider a very simple model: a spinless interacting two-level Anderson model (2LAM), consisting of a lower and an upper QD level $\left(\epsilon_{\ell}, \epsilon_{u}\right)$ with level spacing ${ }^{3} \Delta \equiv \epsilon_{u}-\epsilon_{\ell}$. Analyzing this model using both the numerical renormalization group (NRG) $\stackrel{11}{\underline{1}}$ and a self-consistent Hartree approximation, we study in detail the evolution of the occupation of the single particle levels as a function of gate voltage at $T=0$ for various values of the $\mathrm{QD}$ parameters $(\Gamma, \Delta, U)$. The generic picture of the charging process emerging from this analysis is the following: for any finite coupling to the leads $\Gamma(\nless \Delta)$, sweeping the levels towards the Fermi level of the leads (by tuning the gate voltage) causes the occupations of both the lower and the upper level to increase at comparable rates. This process continues until one level takes over and becomes more occupied than the other. At this point the electron that occupies this level electrostatically repels the other level, pushing up its energy and thereby emptying it. As a result the occupation of the other level performs an oscillation as the gate voltage is swept. The naive QD charging scheme, in which every step of the CB staircase is associated with the filling of only one single particle level in the QD, is only achieved when these charge oscillations are small, i.e. for $\Gamma \ll \Delta$. Below, we discuss in detail the physics of "charge oscillations" and the dependence of their amplitude and form on the system parameters. In particular, we discuss under which conditions the amplitude of these oscillations can be made so large as to cause a significant "occupation inversion", i.e. a situation for which the occupation of the lower level is smaller than that of the upper level. It is important to mention that more general models, e.g. including spin, show effects similar to those described above.

The rest of the paper is organized as follows. In Section II we describe in detail the model we consider. In Section III, we present the result of the NRG calculation for the evolution of the occupation of the two levels as a function of gate voltage [Section III A], and a simple and qualitative understanding of the emerging picture in terms of an Hartree approach [Section III B]. In Section IV, we present a detailed analysis of the phenomenon of occupation inversions, by studying the $2 \mathrm{LAM}$ in the case where the two levels are unequally coupled to the leads. Finally, in Section V we summarize our conclusions, and discuss the robustness of the effects obtained when including spin, and possible experimental tests for our predictions. 


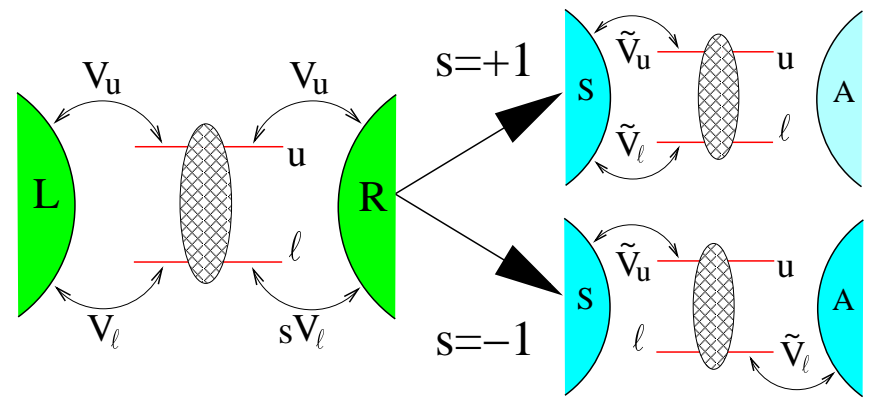

FIG. 1: Schematic depiction of the model. The sign $s$ between the tunneling amplitudes $V_{\ell R}$ and $V_{\ell L}$ determines whether the two dot levels couple to the same (symmetric) channel ( $s=$ +1 , upper mapping), or to two different channels $(s=-1$, lower mapping), with strength $\tilde{V}_{i}=\sqrt{2} V_{i}, i=\ell, u$.

\section{MODEL}

We consider a spinless 2LAM with Hamiltonian $\hat{\mathcal{H}}=$ $\hat{\mathcal{H}}_{d}+\hat{\mathcal{H}}_{l}+\hat{\mathcal{H}}_{l d}$. (The spinful case will be considered briefly in Section $\nabla$ ) Two leads, identical, noninteracting and in equilibrium, are described by $\hat{\mathcal{H}}_{l}=\sum_{k a} \epsilon_{k a} c_{k a}^{\dagger} c_{k a}$, where $c_{k a}^{\dagger}$ creates an electron with energy $\epsilon_{k}$ in lead $a=L, R$. The isolated QD is described by

$$
\hat{\mathcal{H}}_{d}=\sum_{i=u, \ell} \epsilon_{i} d_{i}^{\dagger} d_{i}+U \hat{n}_{\ell} \hat{n}_{u}
$$

where $d_{i}^{\dagger}$ creates an electron in the QD in level $\epsilon_{i}$ $(i=\ell, u)$, measured w.r.t. the Fermi level defined by the leads, $\hat{n}_{i}=d_{i}^{\dagger} d_{i}$ is the number operator, and $U$ is the charging energy, which we fix at $U=0.2 \mathrm{D}$ throughout this paper, $2 D$ being the bandwidth. Finally, the tunneling between the QD and the leads is described by $\hat{\mathcal{H}}_{l d}=\sum_{k i a}\left(V_{k i a} c_{k a}^{\dagger} d_{i}+h . c.\right)$. We consider $k$ independent tunneling matrix elements $V_{k i a}=V_{i a}$, which are $L$ - $R$-symmetric in magnitude ${ }^{4}, V_{u L}=V_{u R}=V_{u}$ and $V_{\ell L}=s V_{\ell R}=V_{\ell}$, but with a possible relative phase $s=\exp (i \phi)$ between the $L$ and $R$ matrix elements of the lower leve $\mathrm{1}^{5,6,7.8}$. Time reversal symmetry implies $\phi=0, \pi$ (hence $s= \pm 1$ ). The corresponding bare level widths are $\Gamma_{i}=2 \pi \rho V_{i}^{2}$, where $\rho$ is the density of states in the leads.

The two possible choices for $s= \pm 1$ lead to two distinct models (see Fig. (1): (i) for $s=+1$, both local levels couple to the same channel, namely the symmetric linear combination of the left and the right lead $\left(c_{k L}+c_{k R}\right)$, while the antisymmetric combination $\left(c_{k L}-c_{k R}\right)$ decouples completely; (ii) for $s=-1$, the upper and lower local levels couple to different channels, namely to the symmetric or antisymmetric combinations, respectively $\stackrel{9}{ }$.

We shall denote the ground state expectation value of the occupation of level $i$ by $n_{i}=\left\langle\hat{n}_{i}\right\rangle$, and parameterize the gate voltage by the average bare level position $\epsilon \equiv\left(\epsilon_{\ell}+\epsilon_{u}\right) / 2$. For $\Gamma_{\ell}=\Gamma_{u}=\Gamma$, this parameterization reveals particle-hole symmetry $\underline{10}$ around $\epsilon^{*}=-U / 2$, namely $n_{u}\left(\epsilon+\epsilon^{*}\right)=1-n_{\ell}\left(\epsilon^{*}-\epsilon\right)$, independent of $\Delta$ and $\Gamma$. If $\Gamma_{\ell} \neq \Gamma_{u}$, this symmetry is broken for both $s= \pm 1$.

\section{CHARGE OSCILLATIONS}

In this section, we study the evolution of $n_{i}(i=1,2)$ as a function of the gate voltage $\epsilon$, and discuss the physical origin of non-monotonic occupation of the two levels. We shall first present the results of our NRG 11 calculations for $n_{i}$, and then use a simple Hartree analysis to gain some insight into the NRG results.

\section{A. NRG calculations}

We start our analysis by considering equally coupled levels, $\Gamma_{\ell}=\Gamma_{u}=\Gamma$, and use the $\mathrm{NRG}^{11}$ to calculate the $\epsilon$-dependence of $n_{i}$. Naively one may expect that if the QD is initially empty, the QD levels get occupied monotonically one by one as $\epsilon$ is decreased, the usual CB behavior. In other words, first the occupation of the lower level would be expected to increase monotonically as $\epsilon_{\ell}$ crosses the Fermi level, and subsequently $n_{u}$ would increase as $\epsilon_{u}+U$ approaches it. However, our NRG results [Fig. 2] show that this intuitive picture is valid only if the coupling to the leads is much smaller than the dot level spacing, $\Gamma \ll \Delta$. In particular, when $\Gamma \gtrsim \Delta$, $n_{\ell}$ and $n_{u}$ show a non-monotonic $\epsilon$-dependence, characterized by charge oscillations of $n_{u}$ (or $n_{\ell}$ ) when the lower (or upper) level crosses the Fermi leve ${ }^{12}$. The oscillation in $n_{u}$ occurs because as soon as the lower level begins to be occupied significantly, the system can gain charging energy by additionally filling the lower level and emptying the upper level (an analogous argument works for $\left.n_{\ell}\right)$.

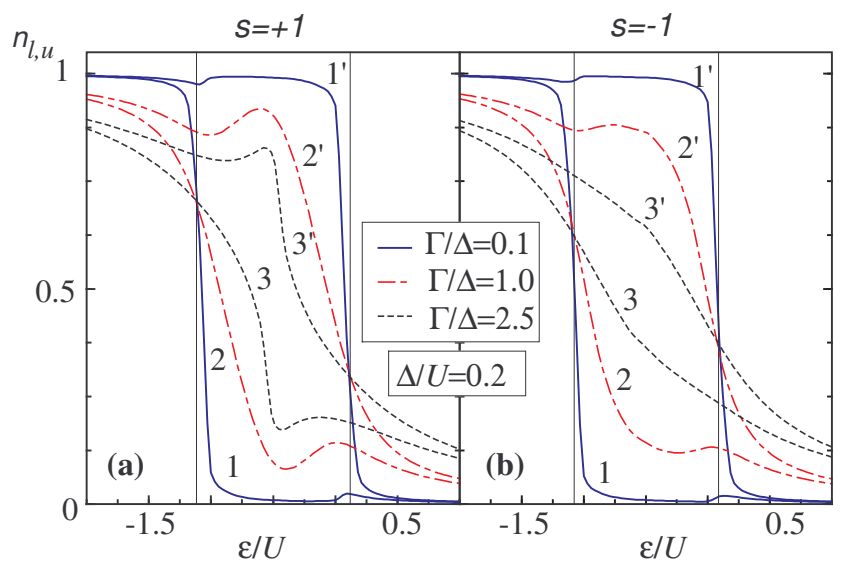

FIG. 2: NRG results for the occupation of the lower $\left[n_{\ell}\right.$, (curves $\left.\left.1^{\prime}-3^{\prime}\right)\right]$ and the upper level $\left[n_{u}\right.$, (curves $\left.\left.1-3\right)\right]$ for fixed $\Delta$ and different values of $\Gamma$, for (a): $s=+1$ and (b): $s=-1$. The dotted lines indicate where the lower/upper level crosses the Fermi level, at $\epsilon=\Delta / 2$ and $\epsilon=-U-\Delta / 2$. 
To explore how strongly these charge oscillations vary with $\Gamma, \Delta$ and how they are affected by the sign of $s$, we show in Fig. 2 the behavior of $n_{i}(\epsilon)$ for variable $\Gamma$ and $s= \pm 1$, keeping $\Delta$ fixed (at $0.2 U)$. In the limit $\Gamma / \Delta \ll 1$ level $\ell$ becomes occupied rather suddenly (curve $1^{\prime}$ ) when it crosses the Fermi level at $\epsilon=\Delta / 2$, and similarly for the upper level at $\epsilon=-U-\Delta / 2$ (curve 1 ). In addition to this typical CB behavior, we observe, even for the smallest $\Gamma$ considered $(\Gamma=0.1 \Delta)$, a tiny non-monotonicity or charge oscillation in $n_{u}\left(n_{\ell}\right)$ roughly at that $\epsilon$ where the occupation of the lower (upper) level increases sharply from 0 to 1 . A gradual increase of $\Gamma$ towards $\Gamma / \Delta \approx 1$ results in a strengthening of these charge oscillations. In the limit $\Gamma / \Delta \gg 1$, Fig. 3(c) and (d), the monotonic increase in the occupation is recovered, though the actual dependence of $n_{i}$ on $\epsilon$ depends strongly on the sign of $s$.

\section{B. Hartree approach}

A simple and qualitative understanding of the charge oscillations observed in our NRG results can be obtained in the framework of a self-consistent Hartree approximation ( $\mathrm{scHA}$ ), by studying the evolution of the Hartree levels as function of $\epsilon$. This scheme accounts for the interaction by replacing the bare levels $\epsilon_{i}$ of the noninteracting problem, with the corresponding Hartree levels

$$
\epsilon_{i} \rightarrow \epsilon_{i}^{H}=\epsilon_{i}+U n_{\bar{\imath}},
$$

where $\bar{\imath}=\ell / u$ if $i=u / \ell$. By integrating out the leads, we obtain the effective noninteracting dot Hamiltonian

$$
\mathcal{H}_{d}^{\mathrm{eff}}=\left(\begin{array}{cc}
\epsilon_{u}^{H}-i \Gamma_{u} & \left(-i \sqrt{\Gamma_{u} \Gamma_{\ell}}\right) \delta_{s,+1} \\
\left(-i \sqrt{\Gamma_{u} \Gamma_{\ell}}\right) \delta_{s,+1} & \epsilon_{\ell}^{H}-i \Gamma_{\ell}
\end{array}\right) .
$$

The corresponding retarded dot Green's function, defined as $\mathcal{G}_{i j}^{R}(t) \equiv-i \theta(t)\left\langle\left\{d_{i}(t), d_{j}^{\dagger}(0)\right\}\right\rangle$, can be obtained exactly for both values of $s$, by solving the matrix equation $\mathcal{G}^{R}(\omega)=\left(\omega-\mathcal{H}_{\text {eff }}\right)^{-1}$. To finally obtain the Hartree approximation for the interacting Green's function, one has to self-consistently calculate the average level occupation $n_{i}(\epsilon)$, using the $T=0$ relation

$$
n_{i}(\epsilon)=-\frac{1}{\pi} \int_{-\infty}^{0} d \omega \operatorname{Im} \mathcal{G}_{i i}^{R}(\omega, \epsilon)
$$

Since the self-consistent Hartree equation (4) may have more than one solution, a criterion is needed to pick the correct one. To this end, we note that, for given $\epsilon$, the system adjusts its local level occupations $n_{u}$ and $n_{\ell}$ such that its total free energy $F_{s}\left(n_{u}, n_{\ell}\right)$ is minimized. Within the scHA approach, $F_{s}$ can be obtained by integrating Eq. (44), so that the conditions for $F_{s}$ to be extremal, $\partial F_{s} / \partial n_{i}=0$, reproduce Eq. (4); we should then pick that solution of Eq. (4) for which the extremum is a global minimum of $F_{s}$. In the case of two nearly degenerate minima, the scHA neglects the possibility of tunneling between them, and a different approach has to be considered. Nevertheless we find, somewhat unexpectedly, that in the case of exactly degenerate minima, e.g. $\Delta=0$, an average over the minima reproduces the NRG results rather accurately [see $\Gamma / \Delta=\infty$ curve in Fig. 31(d)].

For simplicity we implemented this strategy explicitly for $s=-1$, but not for $s=+1$, since for the latter $\mathcal{H}_{d}^{\text {eff }}$ is not diagonal, which makes the determination of $F_{+1}$ very tedious. For $s=-1$, Eq. (4) yields the condition $n_{i}(\epsilon)=$ $\frac{1}{2}-\frac{1}{\pi} \arctan \left\{\left(\epsilon_{i}+U n_{\bar{\imath}}\right) / \Gamma_{i}\right\}$, and the corresponding free energy has the form

$$
F_{-1}=U n_{\ell} n_{u}+\sum_{i=u, \ell}\left[\epsilon_{i} n_{i}-\frac{\Gamma_{i}}{\pi} \log \left(\sin \pi n_{i}\right)\right] .
$$

Fig. 3 compare NRG with corresponding scHA results for $n_{i}(\epsilon)$. For $s=-1$, we minimized $F_{-1}$ [Eq. (5)] and find remarkably good agreement between NRG and scHA. For $s=+1$, for which we did not determine $F_{+1}$, we show the results of a "naive scHA", obtained by simply plotting a numerical solution of Eq. (4) and "hoping" (without checking) that it is the correct one. Clearly, the results so obtained cannot be trusted on their own merit; we present the naive scHA results nevertheless, to illustrate precisely this point: indeed, in Fig. B $3(\mathrm{a}, \mathrm{c})[$ for $\Gamma / \Delta>1]$ they do not agree well with NRG results.

Fig. 31 (c) and (d) include a special situation, namely $\gamma=1$ and $\Delta=0$, for which both $\epsilon_{\ell}=\epsilon_{u}$ and $\epsilon_{\ell}^{H}=\epsilon_{u}^{H}$. This causes a sudden jump for $s=+1$ in $n_{u}$, but none for $s=-1$ [cf. dashed lines in Fig. 3](c) and 3] (d), re-

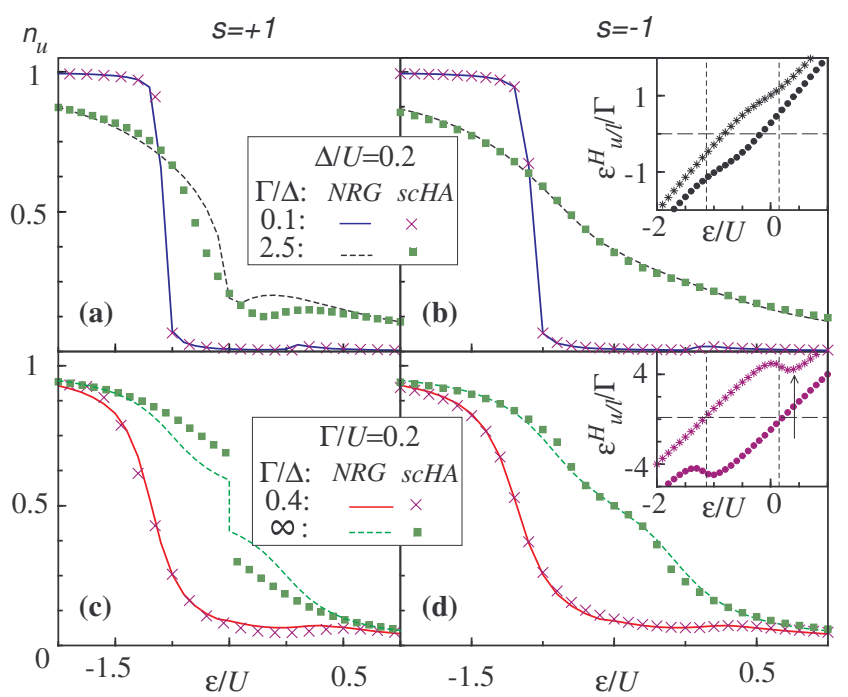

FIG. 3: Comparison of NRG and scHA results for $n_{u}(\epsilon)$, for fixed $\Delta$ and variable $\Gamma[(\mathrm{a})$, (b)] or fixed $\Gamma$ and variable $\Delta$ $[(\mathrm{c}),(\mathrm{d})]$. The naive scHA used for $s=+1$ works well for $\Gamma / \Delta<1$. Insets in (b) and (d): Hartree levels $\epsilon_{\ell}^{H}$ (circles) and $\epsilon_{u}^{H}$ (stars), calculated via the scHA from Eq. (2), for $\Gamma / \Delta=0.1$ in (b) and 0.4 in (d). For $\Gamma / \Delta=\infty$ the Hartree levels are degenerate (not shown). The arrow in inset (d) indicates the value of $\epsilon_{0}$, the local minimum of $\epsilon_{u}^{H}$. 
spectively]. To understand why, note that for $\Delta=0$ and $s=+1$ the odd local combination $\left(d_{u}-d_{\ell}\right)$ decouples from the leads; thus, its width is zero and hence its occupation increases abruptly when its energy drops below zero. On the other hand, for $s=-1$ the occupation increases gradually, since the width of the odd combination is comparable to that of the even one $\left(d_{u}+d_{\ell}\right)$. A similar argument explains why for small but non-zero $\Delta / \Gamma$ (odd level almost decoupled for $s=+1$ ) as in Fig. 2 curves 3 and $3^{\prime}$, the charge oscillations are still observable for $s=+1$ but not for $s=-1$.

\section{UNEQUAL COUPLING AMPLITUDES LEADING TO OCCUPATION INVERSION}

In addition to providing a simple way to compute physical quantities, the scHA, and in particular the concept of Hartree levels [see insets of Fig. 3 (b,d)], may be used to qualitatively understand how the physics of the charge oscillations depends on the various system parameters. Suppose that both Hartree levels are swept downwards, starting from $\epsilon$ well above the Fermi level. When the lower level comes within $\Gamma_{\ell}$ of the Fermi level, it begins to fill up and the upper Hartree level $\epsilon_{u}^{H}$ is pushed up by $U$, causing a charge oscillation in $n_{u}$. The latter will be stronger the larger $n_{u}$ was before the oscillation, i.e., the larger the width $\left(\Gamma_{u}\right)$ of the upper level, and the lower the value $\epsilon_{u}^{H}\left(\epsilon_{0}\right)$ of the upper Hartree level at its local minimum, say $\epsilon_{0}$ (cf. Fig. [3). Indeed, if $\epsilon_{u}^{H}\left(\epsilon_{0}\right) \leq \Gamma_{u}$, then the upper level achieves a rather significant occupation before full occupation of the lower level (and corresponding emptying of the upper one), implying an increase in the amplitude of the corresponding charge oscillation. Moreover, since $\epsilon_{u}^{H}\left(\epsilon_{0}\right)$ is also the lower the more suddenly the lower level gets filled, a smaller $\Gamma_{\ell}$ also strengthens the charge oscillations. Thus, strong charge oscillations can be obtained quite generally by allowing $\gamma \equiv \Gamma_{u} / \Gamma_{\ell} \neq 1$. The above argument implies that $n_{u}$-oscillations are enhanced for $\gamma \gg 1$; by an analogous argument, with $\ell \leftrightarrow u$, $n_{\ell^{-}}$oscillations are strengthened for $\gamma \ll 1$.

It appears that the oscillations are so strong that when $\gamma \neq 1$, the lower and the upper Hartree levels might actually cross each other (see inset Fig. (5), leading to an inverted occupation [Figs. 45] and 6 .

Since the bare energy levels are separated by the level spacing $\Delta$, the conditions $U n \gtrsim \Delta$ and $\max \left(\Gamma_{\ell}, \Gamma_{u}\right) \gtrsim \Delta$ must be met to achieve such an occupation inversion. Figs. 4 to $[6$ show how the asymmetry $(\gamma \neq 1)$ of the couplings affects the occupation of level $\ell$ and $u$ (dashed and solid lines) both for $s= \pm 1$, leading to an inversion of the occupation within a certain range of $\epsilon$.

Our discussion of occupation inversion generalizes a recent related study by Silvestrov and Imry ${ }^{13}$. In an attempt to understand the origin of repeated and abrupt phase lapses observed in the transmission phase of a multilevel QD ${ }^{14}$, they studied a multilevel model consisting of one level (say $u$ with coupling $\Gamma_{u}$ ) strongly coupled

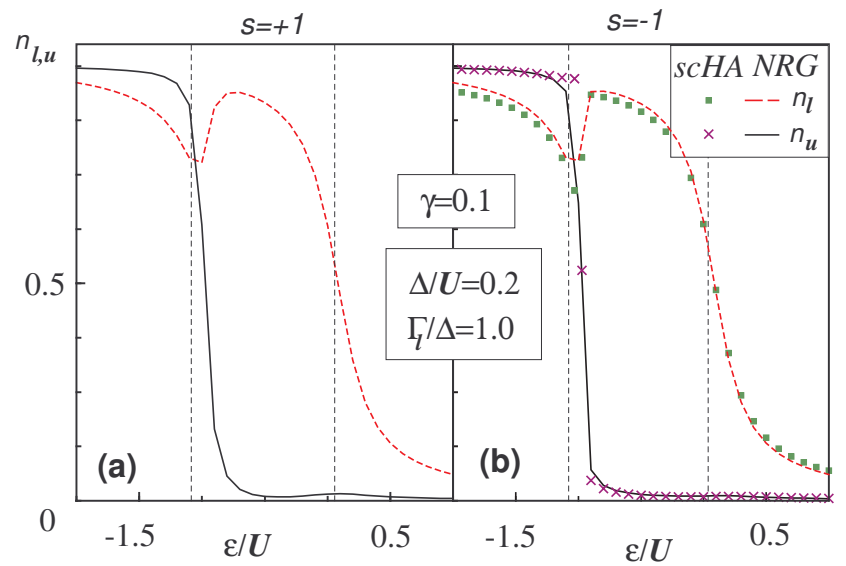

FIG. 4: Allowing $\gamma \equiv \Gamma_{u} / \Gamma_{\ell}$ to be $\neq 1$ results in an inversion of the occupation for a certain range of $\epsilon$. The scHA results ( $n_{u}$ : crosses, $n_{\ell}$ : boxes) for $s=-1$ (where $F_{-1}$ is known) agree well with NRG results $\left(n_{u}\right.$ : solid, $n_{\ell}$ : dashed lines). For $\gamma<1$ the Hartree levels cross near the left CB peak, implying an occupation inversion below the corresponding crossings.

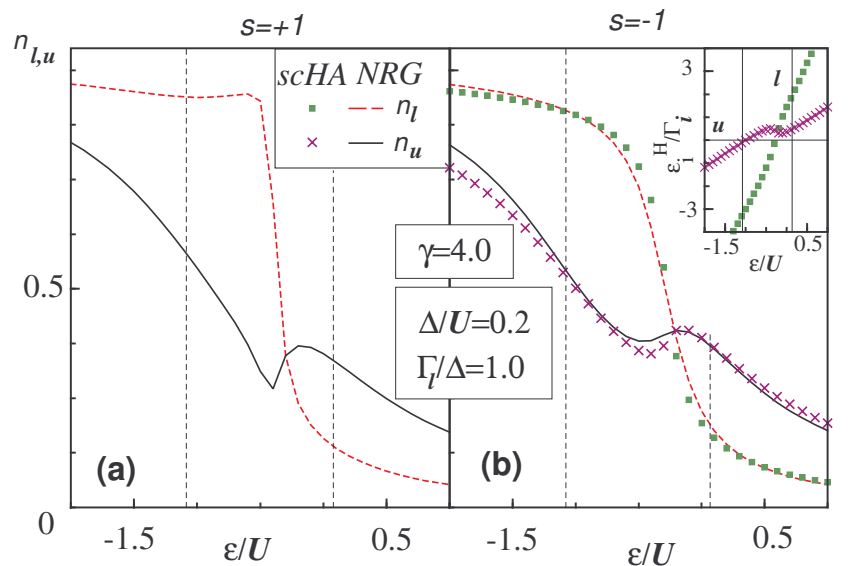

FIG. 5: Same as Fig. 4 but for $\gamma>1$. Now the Hartree levels cross near the right CB peak (see inset (b) for $\gamma=4$ ), implying an occupation inversion above the corresponding crossings. Notice the differences in the shape of the occupation curves between the $s=1$ case and $s=-1$ case.

to the leads and at least one additional weakly coupled level (say $\ell$ with coupling $\Gamma_{\ell}$ ). Ref. 13(a) considered the limiting case $\Gamma_{u} \gg \Delta \gg \Gamma_{\ell} \rightarrow 0$ (Ref. 13(b) also studied finite but small $\Gamma_{\ell}$ and spin), and compared the energies of the configuration $\left(n_{u}, n_{\ell}\right)=(1,0)$ to that of $\left(n_{u}, n_{\ell}\right)=(0,1)$ in second order perturbation theory in the tunneling. Their results indicate that the system is able to sustain an occupation inversion until $\epsilon_{u}=-U /\left[\exp \left(2 \pi \Delta / \Gamma_{u}\right)+1\right] \approx-U / 2$. Although we approach this problem from a different angle, i.e., we either solve it exactly by NRG or first solve the tunnel-coupling exactly and then treat the interaction self-consistently, the inversion range found in Ref. 13 coincides with the 


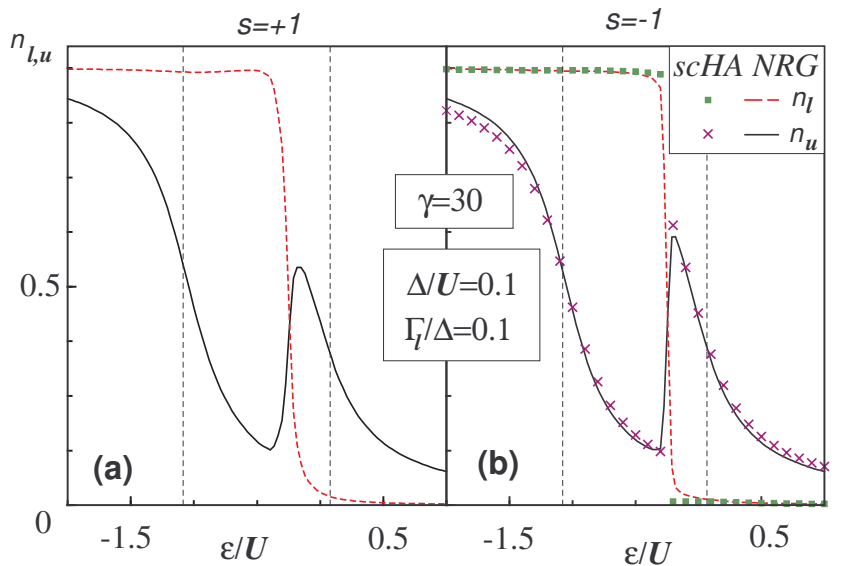

FIG. 6: (a) NRG results for the occupations with $s=1$ and $\gamma=30$. (b) A comparison between the scHA results ( $n_{u}$ crosses, $n_{\ell}$ boxes) and for the NRG results $\left(n_{u}\right.$ solid, $n_{\ell}$ dashed lines), for $s=-1$ (where $F_{-1}$ is known) and $\gamma=30$. The agreement between the NRG and the Hartree approximation is remarkably good and does not depend on $\gamma$

results 15 of this article.

Our analysis indicates that the example of Ref. 13 for occupation inversion is a very special case of a much more general phenomenon whose strength depends on $\gamma$ : as $\gamma$ is increased from 1 (where no occupation inversion occurs), (i) the range of gate voltages in which inversion occurs increases, with the inversion point moving towards the middle of the $\mathrm{CB}$ valley; and (ii) the maximal value reached by $n_{u}$ right before the inversion increases gradually towards 1 , i.e., the effect becomes more pronounced.

\section{SUMMARY}

In this paper, we have studied the gate voltage dependence of the occupation of a spinless two-level Anderson model for the generic case of a relative sign $s$ in the tunneling amplitude. We found a non-monotonic behavior in the occupation of the local levels, due to charging effects between electrons within the QD, and explained this effect in the framework of a self-consistent Hartree approximation. Remarkably, the occupations of the upper and lower levels can even be inverted if the level-to-lead couplings are sufficiently asymmetric. Even though we focused on $T=0$ throughout this paper we expect the calculated behavior to persist as long as $T \lesssim \min \{\Gamma, \Delta, U\}$.

The inclusion of spin in the 2LAM, though making the problem more complex, does not change the qualitative results presented above in a large region of parameter space. To illustrate this, we show in Fig. 7 the total occupation of the upper and lower levels obtained by NRG for the spinful model. The level crossing persists and additional oscillations are observed due to the possibility to put two electrons of opposite spin within each level.

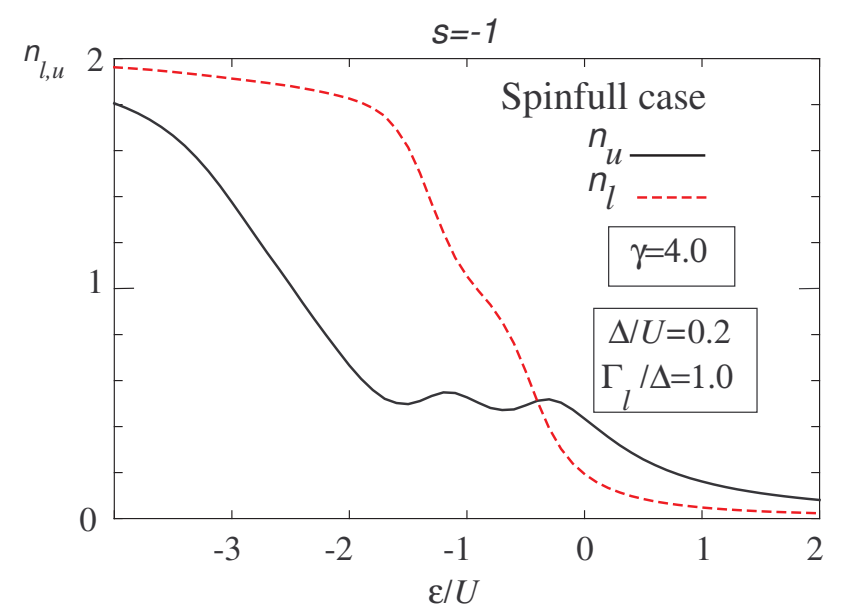

FIG. 7: NRG results in the presence of spin (with the relative sign $s=-1$ ). The parameters are identical to those in Fig. 5] The occupation crossing persists in the spinful case. The additional oscillations in the occupations are observed due to the possibility to put two electrons of opposite spin with in each level.

Our predictions of the non-monotonicity of the charging of a 2LAM are experimentally relevant (in spirit, if not in detail) for any quantum dot system containing orbital levels that are "nearly degenerate", in the sense that their spacing is smaller than the level widths. One way of realizing the specific models studied here would be to use two capacitively coupled quantum dots, each with large level spacings, associating their topmost not-fullyoccupied levels with $\epsilon_{u}$ and $\epsilon_{\ell}$, and using a large magnetic field to lift the Zeeman degeneracy of each. The way in which the charges on these dots evolve with gate voltage, i.e., the evolution of $n_{i}(\epsilon)$, could then be measured experimentally using QPC's serving as extremely sensitive charge sensors, see e.g. Fig. 1 (a) of Ref. 16. In addition, we expect that the occupation of the levels will be reflected in other properties of the system such as the transmission phase. ${ }^{14}$ We leave that, however, for future studies.

\section{ACKNOWELDGEMENTS}

We thank R. Berkovitz, L. Borda, Y. Gefen, U. Hartmann, M. Heiblum, F. Hekking, J. König, F. von Oppen, and G. Zarand for clarifying discussions. Financial support from SFB 631 of the DFG, CeNS, Minerva, DIP c-7.1 and ISF 845/04 is gratefully acknowledged. While performing this work, we became aware of parallel work ${ }^{17}$ that is closely related to ours. 
* Correspondence to michael.sindel@physik.lmu.de

1 U. Meirav and E. B. Foxman, Semicond. Sci. Technol., 10, 255 (1995).

2 D. Loss and D. P. DiVincenzo, Phys. Rev. A 57, 120 (1998).

3 In GaAs-AlGaAs, a $\mathrm{QD}$ of area $A \approx 0.1 \mu \mathrm{m} \times 0.1 \mu \mathrm{m}$ has $\Delta \approx 0.5 \mathrm{meV} \approx 6 \mathrm{~K}$ and $U \approx 1.5 \mathrm{meV}$.

${ }^{4}$ For $V_{u R}=V_{\ell L}=0$, our model includes the case of two dots (levels $u, \ell$ ) separately coupled to two leads $(L, R)$.

5 A. Silva, Y. Oreg, and Y. Gefen, Phys. Rev. B 66, 195316 (2002), study an equivalent noninteracting model for $\left|V_{i a}\right| \equiv V, i=u, \ell, a=L, R$.

6 O. Entin-Wohlman, C. Hartzstein, and Y. Imry, Phys. Rev. B 34, 921 (1986).

7 Q. Sun and T. Lin, Euro. Phys. J. B 5, 913 (1998).

8 D. Boese, W. Hofstetter, and H. Schoeller, Phys. Rev. B 64, 125309 (2001).

9 If we distinguish the symmetric and antisymmetric combination of the leads with an isospin index, the case $s=-1$ corresponds to an effective single-level Anderson model for (iso) spinful electrons, with (iso)spin-dependent couplings and an effective magnetic field $\Delta$; a model used to study a spinful QD coupled to ferromagnetic leads [J. Martinek et al., Phys. Rev. Lett. 91, 247202 (2003)].

${ }^{10}$ For $\Gamma_{\ell}=\Gamma_{u}$ (and both $s= \pm 1$ ), the system is particle-holesymmetric under $c_{k} \rightarrow \tilde{c}_{-k}^{\dagger}$ and $d_{i} \rightarrow-\tilde{d}_{\bar{i}}^{\dagger}$ where $\bar{\imath}=\ell / u$ if $i=u / \ell$, implying $n_{i} \rightarrow\left(1-\tilde{n}_{\bar{i}}\right)$.

11 K. G. Wilson, Rev. Mod. Phys. 47, 773 (1975). We used a flat, symmetric band, discretization parameter $\Lambda=3$ and kept 1024 states.

12 After completion of this work, we became aware that small oscillations had been noted (but not studied systematically) by R. Berkovits, cond-mat/0306284

13 (a) P. G. Silvestrov and Y. Imry, Phys. Rev. Lett. 85, 2565 (2000); (b) P. G. Silvestrov and Y. Imry, Phys. Rev. B 65, 035309 (2002).

14 R. Schuster et al., Nature 385, 417 (1997).

15 The condition $F_{-1}(0,1)=F_{-1}(1,0)$ determines this range.

16 D. Sprinzak, Y. Ji, M. Heiblum, D. Mahalu, and H. Shtrikman, Phys. Rev. Lett. 88, 176805 (2002).

17 J. König and Y. Gefen, cond-mat/0408691 